\title{
Linking the Input to the Output: New Sets of Neurons Complement the Polarization Vision Network in the Locust Central Complex
}

\author{
Stanley Heinze and Uwe Homberg \\ Fachbereich Biologie, Tierphysiologie, Philipps-Universität Marburg, D-35032 Marburg, Germany
}

\begin{abstract}
Polarized light is a key feature of the blue sky, used by many animals as a sensory cue for compass navigation. Like other insects, locusts perceive the $E$-vector orientation of polarized light with a specialized region of their compound eye, the dorsal rim area. Neurons in the brain relay this information through several processing stages to the central complex. The central complex has a modular neuroarchitecture, composed of vertical columns and horizontal layers. Several types of central-complex neurons respond to dorsally presented, rotating $E$-vectors with tonic modulation of their firing frequency. These neurons were found at the input stage of the central complex, as well as near the proposed output stage, where neurons are tuned to form a compass-like representation of $E$-vector orientations underlying the columnar organization of the central complex. To identify neurons suited to link input and output elements, we recorded intracellularly from 45 neurons of the central complex. We report several novel types of polarization-sensitive neurons. One of these is suited to fill the gap between input and output stages of the central-complex polarization vision network. Three types of neurons were sensitive to polarized light in only $50 \%$ of experiments suggesting that they are recruited to the network depending on behavioral context. Finally, we identified two types of neurons suited to transfer information toward thoracic motor circuits. The data underscore the key role of two subunits of the central complex, the lower division of the central body and the protocerebral bridge, in sky compass orientation.
\end{abstract}

\section{Introduction}

Many animals rely on a sky compass for spatial orientation during seasonal migration or homing. A major source of sky compass information is the polarization pattern of the blue sky (Labhart and Meyer, 2002; Horváth and Varjú, 2004). As a result of sunlight scattering, light from the blue sky is partially polarized with celestial $E$-vectors arranged along concentric circles around the sun. The significance of sky polarization for spatial orientation has been established for honeybees (von Frisch, 1949) and desert ants (Wehner, 1984), but orientation responses to dorsally presented polarized light have been demonstrated also in flies, crickets, beetles, and locusts (Wehner and Labhart, 2006). Like other insects, locusts perceive polarized light with a specialized area of their compound eye, the dorsal rim area (Mappes and Homberg, 2004). The dorsal rim area faces the sky and is equipped with photoreceptors of high polarization sensitivity (Eggers and Gewecke, 1993; Homberg and Paech, 2002). Photoreceptor axons project to dorsal areas in the lamina and medulla. Further processing stages of the polarization vision system include the anterior lobe of the lobula, the anterior optic tubercle,

Received Jan. 19, 2009; revised March 10, 2009; accepted March 14, 2009

This work was supported by Deutsche Forschungsgemeinschaft Grant H0 950/16-2. We are grateful to Dr. Keram Pfeiffer for providing Spike2-scripts for data analysis and to Dr. Keram Pfeiffer, Stephan Gebhardt, and Sascha Gotthardt for contributing to physiological data on CL1 cells. We thank Sebastian Richter and Manfred Peil for constructing the stimulation devices and control equipment and Karl Heinz Herklotz for raising desert locusts.

Correspondence should be addressed to Uwe Homberg, Fachbereich Biologie, Tierphysiologie, PhilippsUniversität Marburg, D-35032 Marburg, Germany. E-mail: homberg@staff.uni-marburg.de.

D01:10.1523/JNEUROSCI.0332-09.2009

Copyright $\odot 2009$ Society for Neuroscience $\quad$ 0270-6474/09/294911-11\$15.00/0 the lateral accessory lobe (LAL), and the central complex (Homberg, 2004).

The central complex is a group of neuropils in the center of the protocerebrum. It consists of the protocerebral bridge $(\mathrm{PB})$, the upper and lower divisions of the central body (CBU, CBL, respectively), and the paired noduli (Williams, 1975). The central complex has a unique modular neuroarchitecture, characterized by 16 vertical columns and several horizontal layers in the central body (Heinze and Homberg, 2008). The arrangement of layers and columns is generated by columnar and tangential neurons (Heinze and Homberg, 2008). Three types of tangential neuron connect two small areas of the LALs, the lateral triangle and the median olive, to the CBL (Müller et al., 1997). These neurons are highly sensitive to the $E$-vector orientation of dorsally presented polarized light and respond to a rotating polarizer with tonic modulation of their firing rate (Vitzthum et al., 2002). Each cell is maximally excited when stimulated with a particular, preferred $E$-vector $\left(\Phi_{\max }\right)$, and is maximally inhibited at an $E$-vector $90^{\circ}$ different from $\Phi_{\max }$ (polarization opponency). Electron microscopy showed that these tangential cells are input neurons to the CBL (Müller, 1997), but their postsynaptic partners have not been identified yet.

At another level of the central complex, the PB, tangential and columnar cells form an ordered array of E-vector orientation columns (Heinze and Homberg, 2007). Neurons connecting the polarization-vision pathway from the CBL to the $\mathrm{PB}$ have, however, not been identified. In the present study we characterize two novel types of polarization-sensitive (POL) neuron in the central complex, one of which is a likely candidate for linking the CBL 

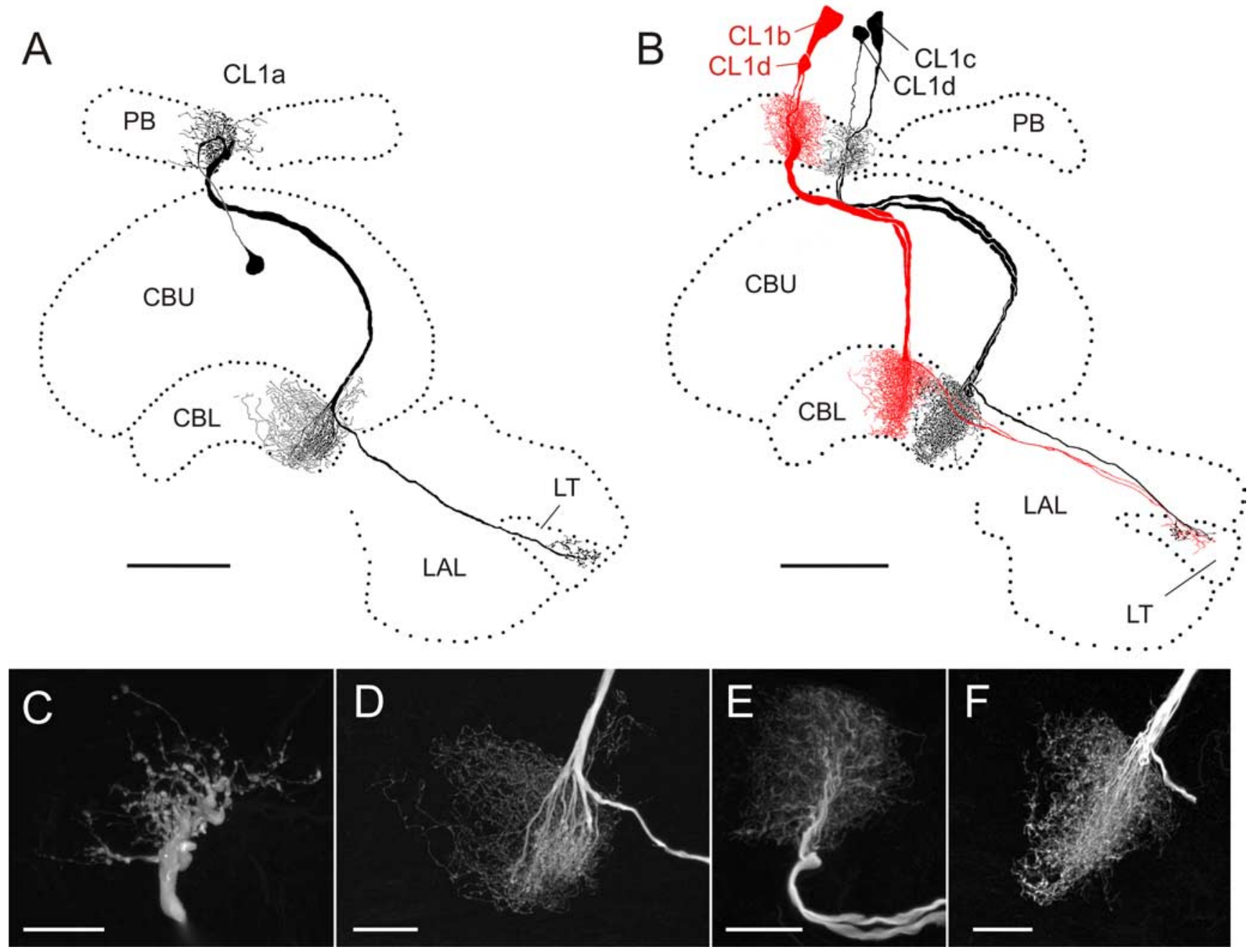

Figure 1. Morphology of CL1 neurons. A, Frontal reconstruction of a CL1a neuron. It connects the CBL with the PB and the LT in the LAL. B, Frontal reconstruction of a CL1b (red) and a CL1c (black) neuron. Each neuron is joined by a fourth type of CL1 neuron, termed CL1d, with arborizations in the same column, but much smaller soma size. CL1c cells lack the additional axonal process to the LT. C, D, Details of arborization trees from the CL1a neuron shown in $\boldsymbol{A}$. Maximal-intensity projections of confocal image stacks reveal varicose endings in the PB $(\boldsymbol{C})$ and a center-surround organization of the arborization tree in the CBL (D). CBL arborizations are of varicose appearance in the center, surrounded by smooth, fine processes in the periphery. $\boldsymbol{E}$, $\boldsymbol{F}$, Details of arborizations of the $\mathrm{CL} 1 \mathrm{c} / \mathrm{d}$ neurons shown in $\boldsymbol{B}$. Confocal images show that the polarity of these cells is reversed compared with that of CL1a neurons. Arborizations in the PB are of smooth appearance $(\boldsymbol{E})$, whereas uniformly varicose endings are present in the $\mathrm{CBL}(\boldsymbol{F})$. Scale bars: $\boldsymbol{A}, \boldsymbol{B}, 80 \mu \mathrm{m} ; \boldsymbol{C}-\boldsymbol{F}, 20 \mu \mathrm{m}$.

inputs to the PB. Three additional types of neuron were polarization sensitive in $50 \%$ of the recordings, suggesting state dependent recruitment to the core polarization vision network. Finally, we identified candidate elements connecting the polarization vision network of the central complex to descending pathways.

\section{Materials and Methods}

Preparation of animals. Locusts (Schistocerca gregaria) were raised in crowded colonies at the University of Marburg in a $12 \mathrm{~h}$ light/dark photoperiod at constant temperature $\left(28^{\circ} \mathrm{C}\right)$. Adult animals of both sexes were used 1-3 weeks after imaginal moult. They were cold anesthetized at $4^{\circ} \mathrm{C}$ for 15-30 $\mathrm{min}$ and were waxed onto a metal holder. In some preparations animals were fixed to the holder with tape for increased stability. Legs and wings were removed and stumps were sealed with wax or glue to prevent hemolymph leakage. The head capsule was opened frontally and the brain was freed of surrounding trachea and fat body. Most muscles in the head capsule were transected. In most preparations, antennal nerves were cut and antennae were removed. To increase stability, the esophagus was transected and the gut was removed from the opened abdomen, which was resealed either using petroleum jelly or a tightly knotted thread. The animal was then mounted either in a horizontal or vertical orientation in the recording setup. For upright mountings, the brain was supported with a metal holder inserted ventrally. For horizontal mountings, the brain was supported with a wax/wire platform, which was inserted ventrally and waxed to the head. The latter preparation left the frontal field of view open for stimulus display. Either the metal support platform or an additional silver wire inserted into the hemolymph was used as reference electrode. To facilitate electrode penetration, the neural sheath was removed mechanically with forceps. Desiccation was prevented by submerging the brain in locust saline (Clements and May, 1974) at all times.

Electrophysiology and stimulation. Intracellular recordings were performed with sharp electrodes (resistance 60-150 M 2 ), drawn from borosilicate capillaries (inner diameter, $0.75 \mathrm{~mm}$; outer diameter, $1.5 \mathrm{~mm}$; Hilgenberg) using a Flaming/Brown horizontal puller (P-97, Sutter). Electrode tips were filled with $4 \%$ Neurobiotin (Vector Laboratories) in $1 \mathrm{~m} \mathrm{KCl}$ and backed up with $1 \mathrm{~m} \mathrm{KCl}$. Intracellular signals were amplified $(10 \times)$ with a custom-made amplifier or a SEC05-LX amplifier (NPI). After sampling at a sampling rate of at least $5 \mathrm{kHz}$ (CED1401 Micro, Cambridge Electronic Design; or Digidata 1322A, Molecular Devices), signals were stored on a PC using Spike2- (Cambridge Electronic Design) or PClamp9 software (Molecular Devices). Digital high pass filtering was applied when necessary to compensate for drifting baseline. After recording, depolarizing current was applied (1-3 nA, 1-5 min) to iontophoretically inject Neurobiotin. 
A

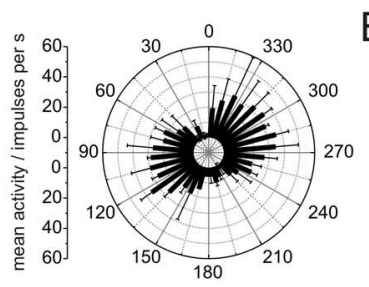

B

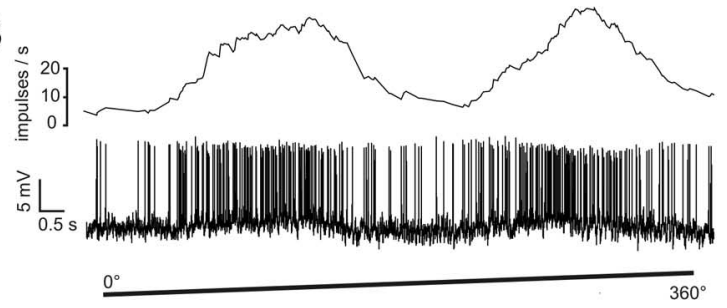

C
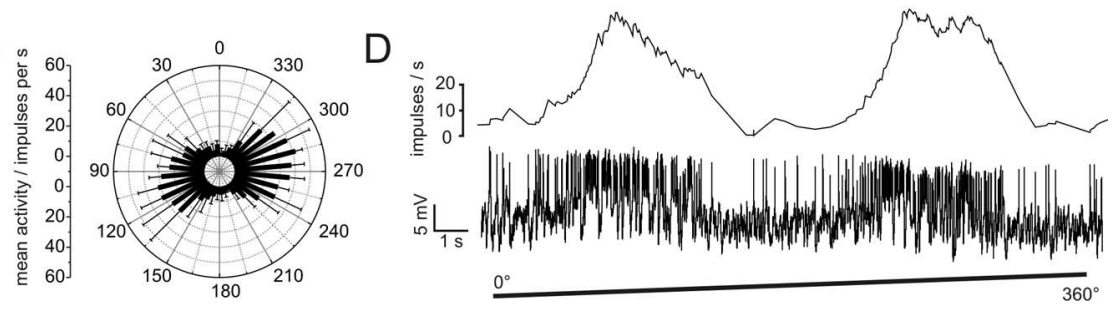

Figure 2. Responses of $\mathrm{CL} 1 \mathrm{a}$ and $\mathrm{CL} 1 \mathrm{~b} / \mathrm{d}$ neurons to polarized light. $A$, Circular diagram of mean frequencies of action potentials of a CL1a neuron plotted against $E$-vector orientation during dorsal stimulation with a rotating polarizer $(n=2$, error bars $=$ SD, bin size $10^{\circ}, p<10^{-12}$ ). $\boldsymbol{B}$, Spike train (lower trace) and mean spiking frequency (upper trace, gliding average with bin size of 1 s) of the same neuron during a clockwise $360^{\circ}$ rotation of the polarizer. C, Mean activity of a double-labeled CL1b/d neuron from four $360^{\circ}$ rotations of the polarizer (error bars, SD; bin size $10^{\circ} ; p<10^{-12}$ ). D, Spike train and mean spiking frequency from the same neuron as in $\mathbf{C}$ during a clockwise $360^{\circ}$ rotation of the polarizer. Postsynaptic potentials are visible throughout the recording.
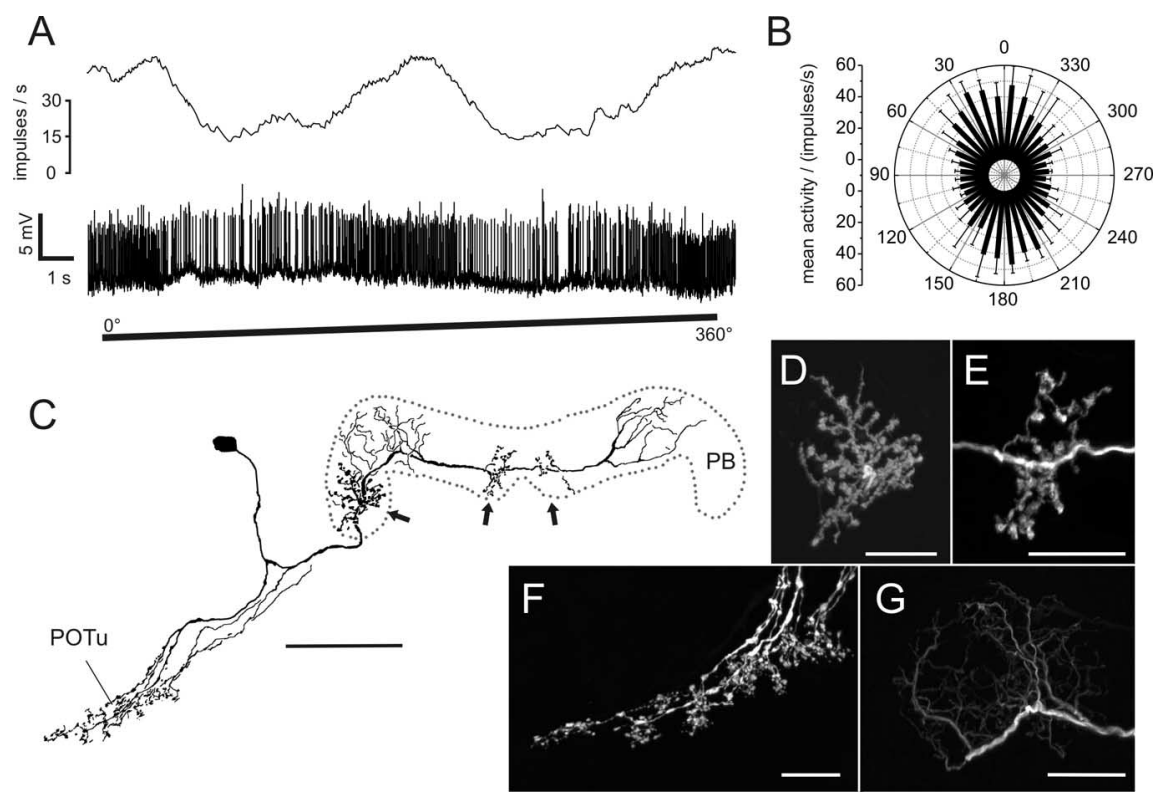

Figure 3. Physiology and morphology of a TB2 neuron. A, Mean activity and intracellular recording trace during clockwise rotation of the polarization filter. $\boldsymbol{B}$, Mean activity of the neuron during $360^{\circ}$ rotations of the polarizer plotted against $E$-vector orientation $\left(n=4\right.$; error bars, SD; bin size $10^{\circ} ; p<10^{-12}$ ). C, Frontal reconstruction of the neuron. Three domains of varicose arborizations (arrows) and two wider regions of smooth arborizations are present in the PB. Ramifications in the posterior optic tubercle (POTu) are also varicose. $\boldsymbol{D}, \boldsymbol{E}$, Detailed views of varicose ramifications in the PB (D, lateral arborizations; $\boldsymbol{E}$, medial arborizations in left hemisphere; maximal-intensity projections of confocal image stacks). $\boldsymbol{F}$, Varicose endings in the POTu (maximal-intensity projection). G, Smooth endings in left hemisphere of the PB (maximal-intensity projection). Scale bars: $C, 80$ $\mu \mathrm{m} ; \mathbf{D}-\mathbf{G}, 20 \mu \mathrm{m}$

For visual stimulation, two different experimental setups were used. The first setup (using vertical mounting of the animal) used a xenon-arc (XBO $150 \mathrm{~W}$ ) as light source. It was connected via a light guide (Schölly; spectral range $400-800 \mathrm{~nm}$ ) to a perimeter placed around the animal. A polarizer (HN38S, Polaroid) or a neutral density filter of equal transmission could be placed in the light path (irradiance at the animal's head: 94 $\mu \mathrm{W} / \mathrm{cm}^{2}$; angular size: $2.7^{\circ}$ ). The second setup was equipped with a halogen bulb as light source $(150 \mathrm{~W})$ to present polarized light from dorsal direction (irradiance: $57 \mu \mathrm{W} / \mathrm{cm}^{2}$ angular size: $3.4^{\circ}$ ). Again, the polarizer could be interchanged with a neutral density filter to present unpolarized light. The horizontal mounting of the locust in this setup allowed us to present unpolarized stimuli in the frontal visual field using a cathode ray tube (CRT)-screen (Mitsubishi, Diamond Pro2070SB, frame rate: $140 \mathrm{~Hz}, 38 \mu \mathrm{W} / \mathrm{cm}^{2}$, angular size: $\left.83^{\circ} \times 110^{\circ}\right)$. The CRT-screen was controlled by a ViSaGe (Cambridge Research Systems), programmed via MatLab. In both setups, the polarizer was rotated via custom built hard- and software by $360^{\circ}$ counterclockwise or clockwise at speeds of $21 \%$ or $30 \%$. An $E$-vector orientation parallel to the longitudinal axis of the animal was defined as $0^{\circ}$.

Data analysis. Sampled spike trains were stored on PC and evaluated using Spike2software with implemented, custom designed scripts. Action potentials were detected with threshold based event detection. The detected events could be visualized as mean frequency using a gliding average algorithm at a bin size of $1 \mathrm{~s}$ or as instantaneous frequency. Background frequency and maximal instantaneous spike frequency were estimated by moving horizontal cursors to the desired level in the respective channel and reading out the current values of the cursor position.

To quantify responses to polarized light, events within each $360^{\circ}$ rotation of the polarizer were assigned a corresponding $E$-vector angle. This list of angles was exported for each rotation and the combined rotations of each neuron were tested for polarization sensitivity. A neuron was rated polarization sensitive if the distribution of angles during filter rotations was significantly different from randomness (Rayleigh test for axial data; Oriana 2.05a; Kovach Computing Services). The mean angle of the distribution was defined as the $\Phi_{\max }$ value of that neuron.

The distributions of $\Phi_{\max }$ values of neuronal populations were evaluated using Rao's spacing test for axial data, which allows very small sample sizes (Batschelet, 1981). To test for correlation of $\Phi_{\max }$ values with the innervated $\mathrm{PB}$ columns, $\Phi_{\max }$ values (ranging from $0^{\circ}-180^{\circ}$ ) were plotted against the innervated columns, separately for each hemisphere or combining both hemispheres. As $\Phi_{\max }$ values are axial data with a periodicity of $180^{\circ}$, data points near the $0^{\circ} / 180^{\circ}$ boundary were shifted by adding $180^{\circ}$ or by subtracting $180^{\circ}$ in the two inner- and outermost columns, respectively. The resulting data sets (ranging from $-20^{\circ}$ to $200^{\circ}$ ) were tested with linear regression analysis for correlation between the $\Phi_{\max }$ value and the innervated PB column ( $t$ test against a regression line with slope of 0 at significance level of 0.01 ; Origin 6.0; Microcal). Correlation coefficients $(R)$ were calculated with the same data sets in Origin 6.0.

Histology. After injection of Neurobiotin, brains were dissected out of the head capsule, cleaned of fat and trachea, and fixed overnight at $4^{\circ} \mathrm{C}$ in Neurobiotin fixative (4\% paraformaldehyde, $0.25 \%$ glutaraldehyde, $2 \%$ saturated picric acid, in $0.1 \mathrm{~m}$ phosphate buffer). Brains were then either processed as whole mounts (see below) or embedded in albumin-gelatin $(4.8 \%$ gelatin and $12 \%$ ovalbumin in demineralized water) for sectioning. Embedded brains were fixed in $8 \%$ formaldehyde (in $0.1 \mathrm{~m}$ phosphate buffer) overnight $\left(4^{\circ} \mathrm{C}\right.$ ), and hereafter sectioned with a vibrating blade microtome at $40 \mu \mathrm{m}$ thickness (VT 
1000S; Leica). Sections were rinsed $3 \times 15 \mathrm{~min}$ in $0.1 \mathrm{M}$ phosphate buffer and incubated with horseradish-peroxidase-conjugated Streptavidin (1:300, Amersham Buchler) in $0.1 \mathrm{M}$ PBS/ $0.5 \%$ Triton X-100 overnight at room temperature. After rinsing in PBS $(3 \times 15 \mathrm{~min})$ and once in $0.05 \mathrm{M}$ Tris/ $\mathrm{HCl}$ ( $15 \mathrm{~min}$ ), diaminobenzidine $\left(0.3 \mathrm{mg} / \mathrm{ml} 3,3^{\prime}\right.$-diaminobenzidine tetrahydrochloride, $0.3 \%$ nickel ammonium sulfate in $0.05 \mathrm{M} \mathrm{Tris} / \mathrm{HCl}$ ) was added, and the reaction was started by adding $0.015 \% \mathrm{H}_{2} \mathrm{O}_{2}$. The staining reaction was stopped by rinsing with $0.05 \mathrm{M}$ Tris/ $\mathrm{HCl}(4 \times 15 \mathrm{~min})$ after the desired intensity was reached. Stained sections were mounted on gelatin/chrome alum coated microscope slides, dehydrated in an increasing ethanol series (50, 70, 90, 95, and 100\%; 3 min each), and cleared in xylene (5 min). Finally, sections were embedded in Entellan (Merck). Reconstructions from diaminobenzidinestained preparations were performed using a camera-lucida attachment on a Leitz compound microscope equipped with a $40 \times$ objective. Drawings were scanned and optimized for contrast and brightness in Adobe Photoshop CS2 (Adobe Systems).

Whole-mount preparations were rinsed $4 \times$ $15 \mathrm{~min}$ in PBS after fixation, and directly incubated with Cy3-conjugated Streptavidin (Dianova; $1: 1000$ ) for $3 \mathrm{~d}$ at $4^{\circ} \mathrm{C}$ in PBT (PBS, including $0.5 \%$ Triton $\mathrm{X}-100)$. After rinsing with PBT $(2 \times 30 \mathrm{~min})$ and PBS $(3 \times 30 \mathrm{~min})$, the brains were dehydrated with an increasing ethanol series (25, 50, 70, 90, 95, and 100\%; $15 \mathrm{~min}$ each), transferred to a fresh mixture of ethanol and methylsalicylate (1:1) for $15 \mathrm{~min}$, and finally cleared in methylsalicylate for $35 \mathrm{~min}$. Brains were eventually mounted in Permount (Fisher Scientific) between two coverslips. To avoid compressions, reinforcement rings were used as spacers. Neurons were scanned with a confocal microscope (Leica TCS-SP2, minimal pinhole size, at $3 \mu \mathrm{m}$ intervals) with a $10 \times$ oilimmersion objective (HC PL APO $10 \times / 0.4$ Imm Corr CS; Leica). Reconstructions based on these data stacks were performed using Adobe Photoshop CS2.

To acquire high resolution data stacks of injected neurons, whole mount preparations were rehydrated and sectioned in thick sections, containing the neuron of interest (for details see Heinze and Homberg, 2008). These preparations were scanned at high resolution $(40 \times$ objective, HCX PL Apo $40 \times / 1.25$ oil) and were reconstructed using Photoshop or shown as maximal projection views as well as single optical sections. All images were optimized for contrast and brightness.

\section{Results}

\section{New sets of POL neurons}

Through intracellular recordings combined with dye fills we identified two sets of POL neurons of the central complex which have not been characterized before. Both types of neuron showed tonic frequency modulations when stimulated with a dorsally rotating polarizer. One type of neuron was identified as CL1 columnar cells (Heinze and Homberg, 2008), whereas the second type was a new class of tangential neurons of the PB, termed TB2.

\section{CL1 neurons}

CL1 neurons have been subdivided into three subtypes (CL1a, CL1b, and CL1c), which differ in details of their arborizations, but share an overall common morphology (discussed in detail in
Heinze and Homberg, 2008). All three cell types have somata in the pars intercerebralis and connect columns of the PB with columns of the CBL via large-diameter fibers (Fig. $1 A, B$ ). Each subtype consists of 16 individual neurons following a precise interhemispheric connectivity scheme (Heinze and Homberg, 2008). CL1a- and CL1b neurons have small-diameter axons projecting to the lateral triangle.

Recordings were obtained from 21 CLla neurons. Recordings from four additional CL1 neurons revealed a CL1b and three CL1c cells which were always colabeled with a fourth type of CL1 neurons, termed CL1d (Fig. $1 B$ ). The neurons were distinguished from each other based on the following characters: First, the size of the soma and primary neurite was larger in CL1b and c cells than in CL1a and d cells (Fig. $1 A, B$ ). Second, the polarity of CL1a neurons was reversed compared with the double-labeled CL1b/d and, CL1c/d neurons. CL1a cells had varicose endings in the PB (Fig. 1C) and a combination of smooth and varicose endings in the CBL (Fig. 1D). Within the CBL, varicosities were confined to single columns, but ran across all layers, whereas smooth endings were approximately three times as wide and were restricted to dorsal layers, mostly layer 2 (Fig. $1 D$ ). In contrast, the CL1b/d and CL1c/d neurons had smooth endings in the PB (Fig. $1 E$ ) and 
A
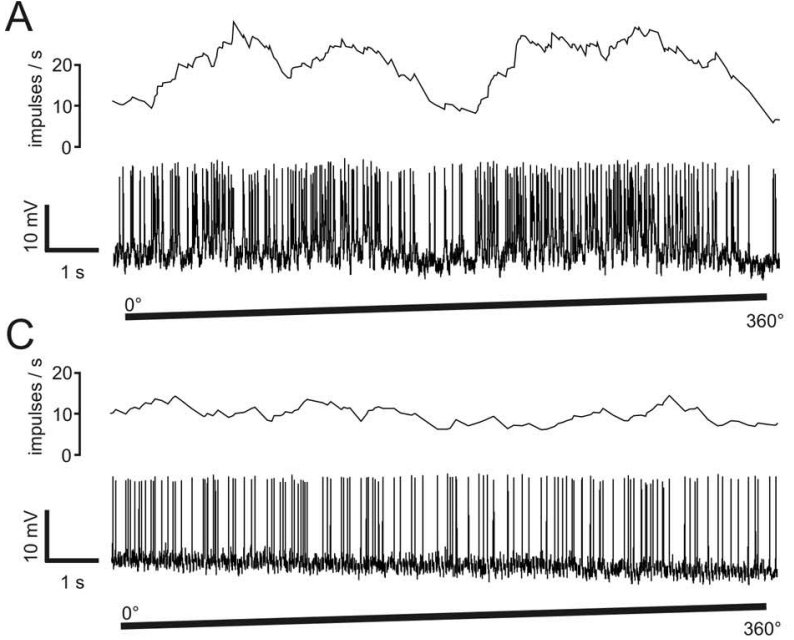

E

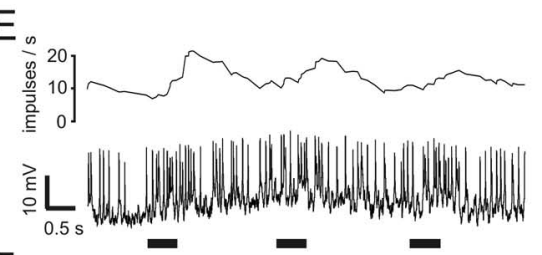

F
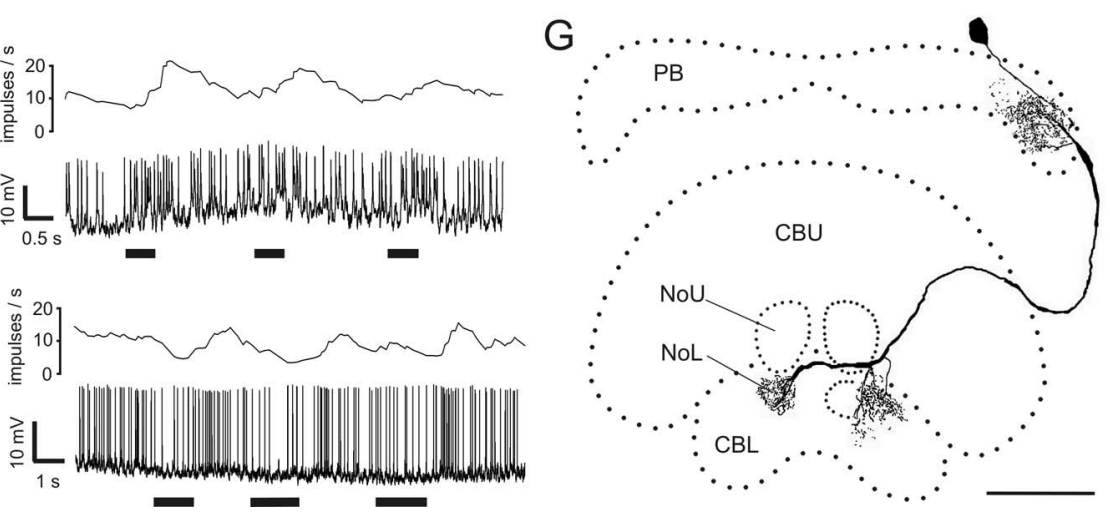

Figure 5. Conditionally polarization-sensitive $\mathrm{CL} 2$ neurons. $\boldsymbol{A}$, Spike train and mean spiking frequency of a polarizationsensitive $\mathrm{CL} 2$ neuron during stimulation with a rotating polarizer. $\boldsymbol{B}$, Mean response during dorsal stimulation with a rotating polarizer ( $n=4$; bin size $10^{\circ}$; error bars, SD; $p=0.009$ ). C, Activity of a polarization-insensitive CL2 neuron during rotation of the polarizer. $\boldsymbol{D}$, Mean spiking frequency from the neuron in $\boldsymbol{C}$ during four rotations of the polarizer (bin size $10^{\circ}$; error bars, SD; $p=$ 0.88). $\boldsymbol{E}, \boldsymbol{F}$, Responses to unpolarized light stimuli (spike trains and mean activity). $\boldsymbol{E}$, The polarization-sensitive neuron (shown in A) shows weak excitation during large-field frontal light flashes. $\boldsymbol{F}$, The polarization-insensitive neuron (shown in $\boldsymbol{C}$ ) responds to small, dorsal flashes of unpolarized light with inhibition followed by weak rebound excitation. $G$, Frontal reconstruction of a $C L 2$ neuron (same as in $\boldsymbol{A}, \boldsymbol{B}$, and $\boldsymbol{E}$ ). The neuron has arborizations in the $\mathrm{PB}$, in the $\mathrm{CBL}$, and in the lower unit of the contralateral nodulus (NoL). NoU, Upper unit of the nodulus. Scale bar, $80 \mu \mathrm{m}$.

exclusively varicose endings in the CBL (Fig. $1 F$ ). Independently of the cell type, endings in the lateral triangle were always varicose. Finally, CL1c cells were defined by the absence of an axon to the lateral triangle but were otherwise identical to CL1b neurons (Heinze and Homberg, 2008).

All CL1 neurons responded to the dorsally presented, rotating polarizer with an E-vector dependent modulation of spike frequency (Fig. 2). The neurons had a mean background activity of $10.2 \pm 4.6$ impulses per second. They were strongly activated to peak instantaneous frequencies of $57.3 \pm 20.9$ (mean \pm SD) impulses per second at their preferred $E$-vector orientation, and were usually totally silenced at $\Phi_{\min }$. When cells were recorded from their arborizations in the $\mathrm{PB}$, we observed only small postsynaptic potentials (PSPs) in most cases. The only example of strong PSPs was found for one of the three recordings from CL1b/d neurons (Fig. 2D).

\section{TB2 neurons}

The second type of POL neuron consisted of tangential neurons of the PB, termed TB2 (Fig. 3). Their somata were located posterior-laterally from the $\mathrm{PB}$ in the posterior pars intercerebralis. The neurons had arborizations in the posterior optic tuber- cle and several distinct arborization domains in the $\mathrm{PB}$ (Fig. $3 C-G$ ). In contrast to TB1 neurons (Heinze and Homberg, 2007), TB2 neurons had three arborization domains with varicose endings in the $\mathrm{PB}$. One of these was located in the lateralmost column of the ipsilateral PB hemisphere (R1 or L1), whereas the other two varicose ramifications were located on either side of the midline innervating parts of columns L8 and R8 (Fig. $3 D, E$ ). In both hemispheres of the $\mathrm{PB}$, arborization domains with smooth endings (Fig. 3G) covered approximately three columns and were separated from the varicose endings by at least one column without ramifications (Fig. 3C). Endings in the posterior optic tubercle appeared strongly varicose (Fig. $3 F$ ). All recorded neurons of this type $(n=5)$ were of indistinguishable morphology with somata in the right hemisphere.

TB2 neurons showed marked polarization opponency with highly similar $E$-vector tuning clustered around $178^{\circ}$ (see Figs. $3 B, 7 B)$. TB2 neurons had a considerably larger tuning width than CL1 neurons and a higher mean background activity of $31 \pm 3.6$ impulses per second. Their peak instantaneous spiking frequency during stimulation was $112 \pm 30$ impulses per second (mean $\pm \mathrm{SD}$ ), and hence about twice as high as in CL1 neurons.

\section{Conditionally}

polarization-sensitive neurons

In contrast to the POL neurons described above, we encountered three types of neuron that showed polarization sensitivity in only $50 \%$ of the experiments. To account for this variability, these neurons were termed conditionally polarization sensitive. Polarization sensitivity of these neurons apparently depended on an unknown factor. Differences in daytime of recording, or differences in anatomy did not correlate with the responsiveness for polarized light. All of these cells were columnar neurons and had been anatomically described as CPU2, CPU4, and CL2 neurons (Heinze and Homberg, 2008).

\section{CPU2 neurons}

CPU2 neurons had smooth arborizations in single columns of the $\mathrm{PB}$ and in corresponding columns of the $\mathrm{CBU}$, where all layers were innervated. Large varicosities were present in the ventral shells of both LALs (Fig. 4G). From a total of six recordings, three cells were polarization sensitive. In these three cells polarization sensitivity was pronounced and highly significant (Fig. 4A,B) (Rayleigh test against uniform distribution, $p<10^{-10}$ ) with peak instantaneous spiking frequencies of $125 \pm 30$ impulses per second at $\Phi_{\max }$. In contrast, three CPU2 neurons were polarizationblind (Fig. 4C,D). The polarization-sensitive cells had a mean background activity of $26 \pm 3.6$ impulses per second, whereas the polarization-insensitive cells only showed a mean activity of $12 \pm$ 7.8 impulses per second. All neurons were characterized by large PSPs during and in between stimulations (Fig. 4A,C). 
Stimulations with unpolarized light flashes were performed in five of the six recorded neurons, either from frontal or from dorsal directions. None of the polarization-sensitive cells showed any detectable response to these light flashes (Fig. $4 E$ ), whereas the polarization-insensitive cells were either inhibited in response to dorsal stimuli or were excited in response to frontal stimuli (Fig. 4F).

\section{CL2 neurons}

Four recordings were obtained from CL2 neurons. These cells connect single columns of the PB with columnar arborization domains in the CBL and with ramifications throughout the lower division of the contralateral nodulus (Fig. 5G) (Heinze and Homberg, 2008).

Two of four recordings showed polarization sensitivity with a mean peak instantaneous activity of $46 \pm 19$ impulses per second and broad tuning curves (Fig. $5 A, B)$. The two remaining neurons did not show frequency modulation during stimulation with a rotating polarizer (Fig. $5 C, D)$. As in CPU2 neurons, background spiking activities of the polarizationsensitive neurons were higher $(13.5 \pm 3.5$ impulses per second) than in the polarization-blind cells $(7.5 \pm 2.1 \mathrm{im}$ pulses per second).

Stimulation with unpolarized light stimuli either from frontal or from dorsal directions led to variable weak responses (Fig. 5E,F). One polarization-sensitive neuron was excited by frontally presented unpolarized light flashes, whereas one polarization-blind cell was inhibited by dorsally presented unpolarized light flashes, followed by rebound excitations at stimulus offset. The remaining neurons did not show any response to dorsally presented light flashes.

\section{CPU4 neurons}

Two recorded neurons could be classified as CPU4 neurons. They connect single columns of the $\mathrm{PB}$ with a columnar region in layer three of the CBU and a layer of the upper division of the contralateral nodulus (Fig. 6A) (Heinze and Homberg, 2008). One of the two cells responded with a significant modulation of its firing rate when presented with the rotating polarizer (Fig. 6B,D), whereas the second cell did not show any response (Fig. 6C,E). Both neurons had similar background firing rates of 6 and 10 impulses per second, respectively. Unpolarized stimuli presented in the frontal visual field elicited an excitatory rebound response at stimulus offset in the polarization-insensitive neuron. As in
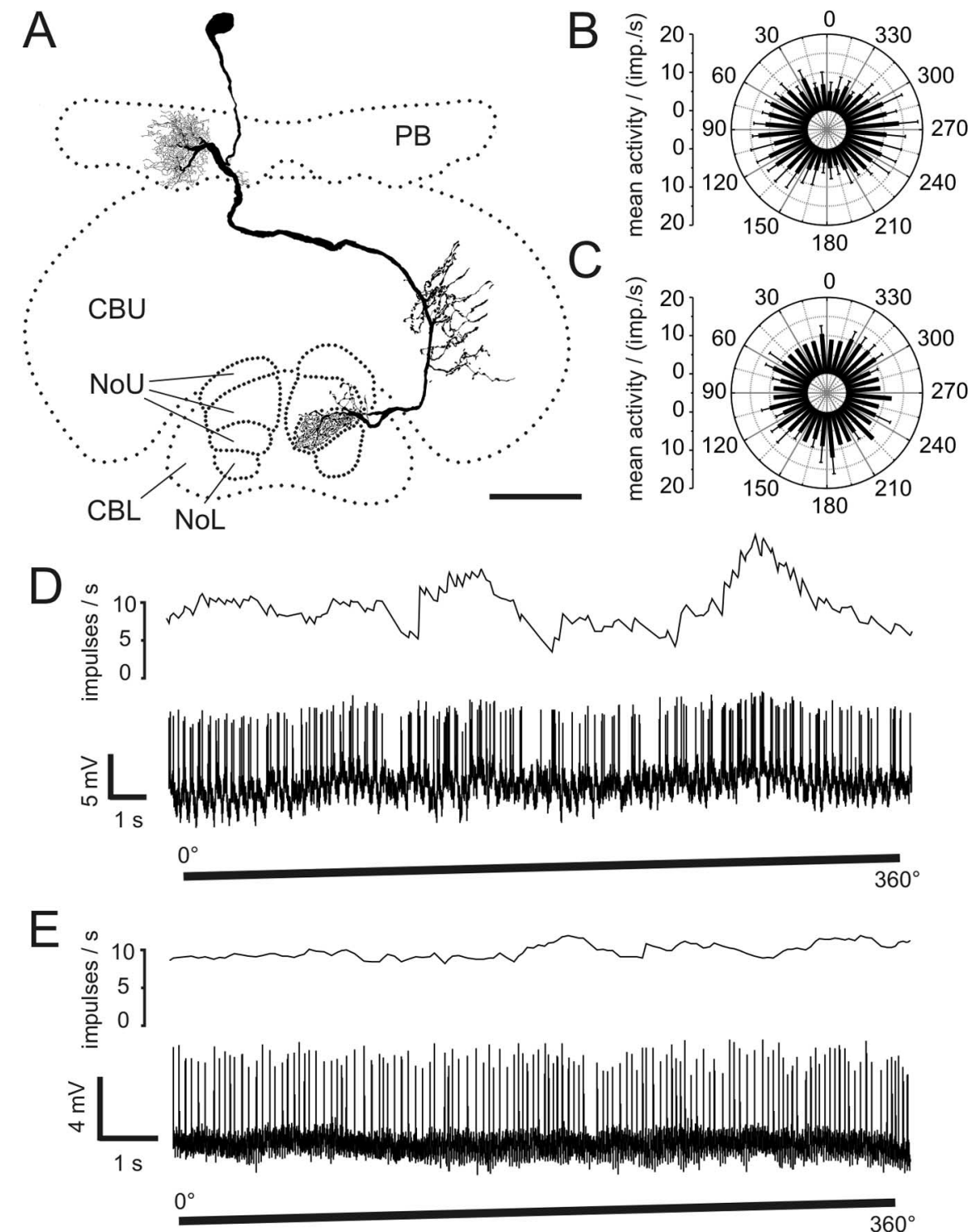

Figure 6. Conditionally polarization-sensitive CPU4 neurons. $A$, Reconstruction of a CPU4c neuron viewed from posterior. Smooth arborizations are present in the PB, whereas endings in the CBU (layer III) and in layer III of the upper unit of the contralateral nodulus ( $\mathrm{NoU}$ ) are varicose. $\boldsymbol{B}$, Mean response of the polarization-sensitive neuron in $\boldsymbol{A}$ during four rotations of the polarizer (bin size $10^{\circ}$; error bars, SD; $p=4.2 \times 10^{-10}$ ). imp., Impulses. $C$, Mean spiking activity (two rotations of the polarizer) of a polarization-blind CPU4b neuron (bin size $10^{\circ}$; error bars, $S D ; p=0.99$ ). $D$, Spiking activity and mean frequency of the CPU4c neuron shown in $\boldsymbol{A}$ during clockwise rotation of the polarizer. $\boldsymbol{E}$, Activity of the (PU4b neuron (same as in $\boldsymbol{C}$ ) during stimulation with a rotating polarizer (spike train and mean frequency). NoL, Lower unit of the nodulus. Scale bar, $50 \mu \mathrm{m}$.
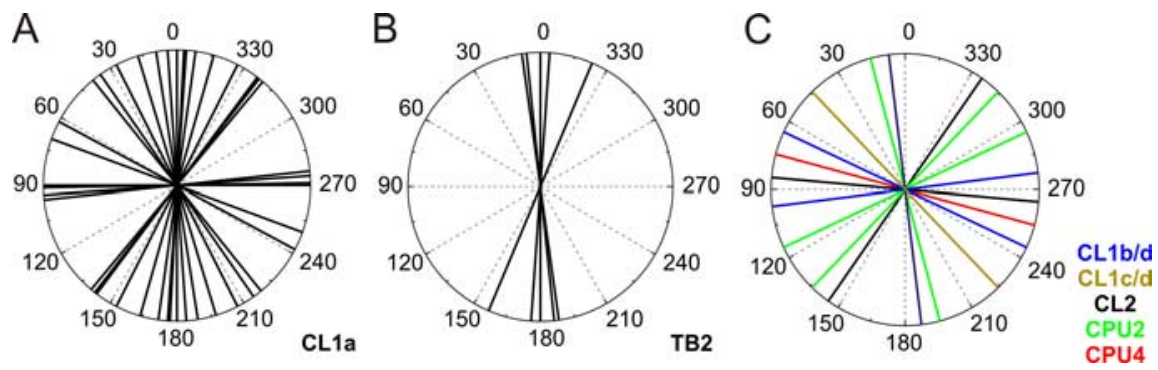

Figure 7. Distribution of $\Phi_{\max }$ values. $\boldsymbol{A}, \Phi_{\max }$ values of CL1a neurons cover the complete range of $180^{\circ}$ with a distribution not significantly different from randomness (Rao's spacing test, $p>0.1$ ). $B, \Phi_{\max }$ values of TB2 neurons are clustered around $178^{\circ}$ (Rao's spacing test, $p<0.01$ ). C, $\Phi_{\max }$ values of all remaining neurons are randomly distributed over the range of $180^{\circ}$ (Rao's spacing test, $p>0.95$ ). 

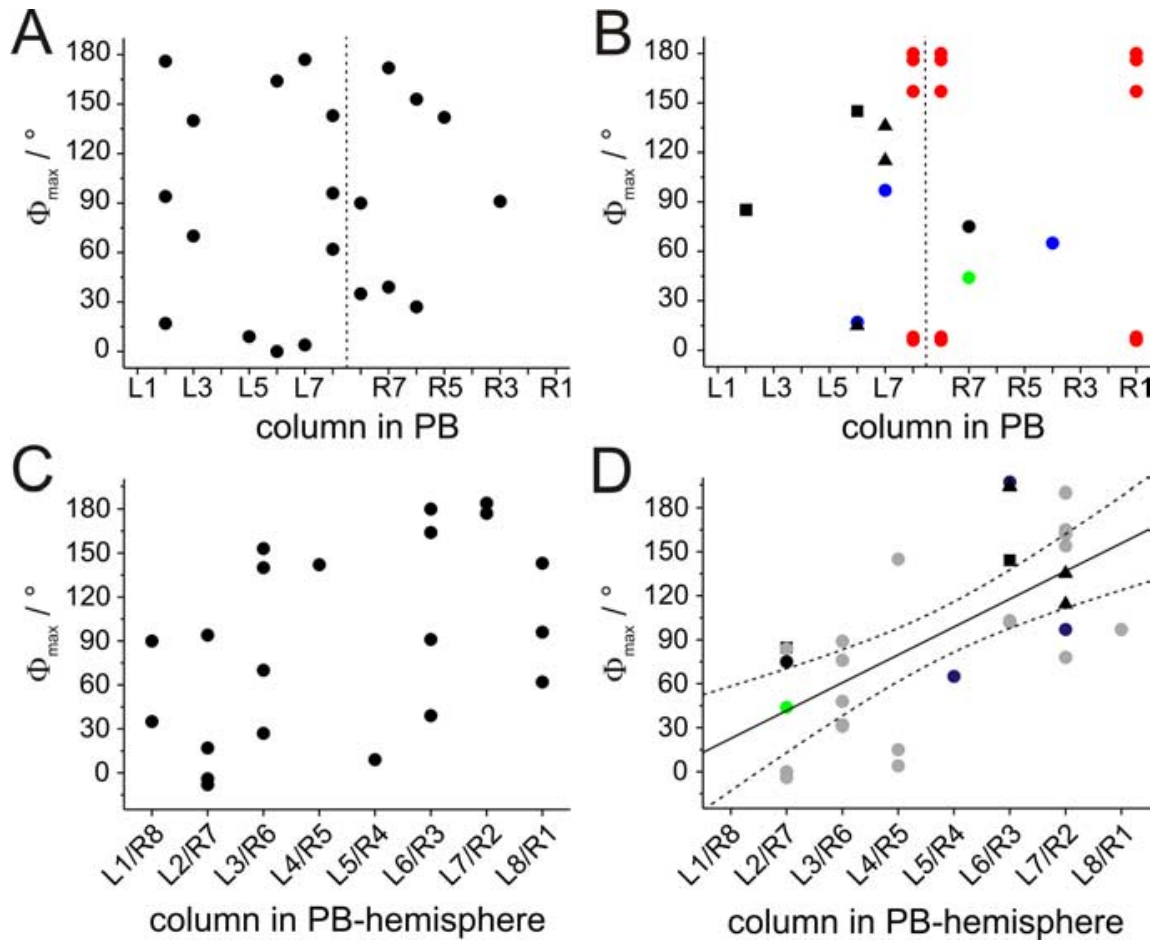

Figure 8. Relation of $\Phi_{\max }$ values to innervated column in the PB. $A, B, \Phi_{\max }$ values plotted against the innervated PB columns. Dashed vertical line indicates the midline of the brain. $\boldsymbol{A}, \mathrm{CL} 1 \mathrm{a}$ neurons. $\boldsymbol{B}, \mathrm{CL} 1 \mathrm{~b} / \mathrm{d}$ neurons (blue), CL1c/d neurons (green), CL2 neurons (black squares), CPU2 neurons (black triangles), CPU4 neurons (black circles), and TB2 neurons (red) C, For linear regression analysis, $\Phi_{\max }$ values of all CL1a neurons were combined in one hemisphere and plotted against the innervated $\mathrm{PB}$ column. Values near the $0^{\circ} / 180^{\circ}$ boundary of $E$-vectors in medial and lateral columns were shifted by adding or subtracting $180^{\circ}$. Within the resulting data set no significant correlation was observed (significance level of $0.01 ; t$ test against slope of $0, p=$ 0.03; correlation coefficient $r=0.47$ ). D, Data from CL1b/d, CL1c/d, CL2, CPU2, and CPU4 neurons (symbols as in B) combined with previously published data from (PU1, CP1, and CP2 neurons (gray circles) (Heinze and Homberg, 2007) result in a highly significant correlation (significance level: $0.01 ; t$ test against slope of $0, p<0.0001, r=0.67 ; y=19 x+3.6$ ).

CPU2 neurons, the polarization-sensitive cell did not respond to unpolarized light presented from dorsally.

The physiological differences in the two recordings from CPU4 neurons may be attributed to the fact that the two cells belonged to different subtypes of CPU4 neurons. The polarization-sensitive cell innervated layer three of the upper division of the nodulus (CPU4c neuron) (Fig. 6A), whereas the polarization-insensitive cell innervated layer two (CPU4b neuron).

\section{Analysis of $\boldsymbol{\Phi}_{\text {max }}$ values}

Heinze and Homberg (2007) showed that E-vector tuning of neurons with arborizations in the $\mathrm{PB}$ corresponds to their columnar arborization domains in the PB in a compass-like manner. We, therefore, asked whether this is also true for the newly presented types of POL neurons.

Data on $E$-vector tuning and columnar arborization domains in the PB were obtained from 21 CL1a neurons. The $\Phi_{\max }$ values of these neurons covered a range of $180^{\circ}$. Their distribution was not significantly different from randomness, despite some apparent clustering (Fig. 7A) (Rao's spacing test, $U=133, p>0.1$ ). The recorded neurons had arborizations in a wide range of columns in the PB. However, when plotting the $\Phi_{\max }$ values of the neurons against their arborization domains in the $\mathrm{PB}$, no obvious correlation was observed (Fig. $8 \mathrm{~A}, \mathrm{C}$ ). Applying linear regression analysis confirmed this result for both hemispheres separately as well as for the combined data $(t$ test for slope $=0$ with $\alpha$-level $=$ 0.01 ; right hemisphere: $p=0.44$, left hemisphere: $p=0.16$, com- bined: $p=0.03$; correlation coefficients $(R): 0.35,0.40$, and 0.47 respectively) (Fig. $8 C)$. As neurons with arborizations in identical columns had different $\Phi_{\max }$ values in different preparations, we wondered whether differences in daytime might be responsible for this observation. Although recording times covered a wide range from 9:45 A.M. to 6:45 P.M., no correlation was observed after plotting $\Phi_{\text {max }}$ values against time of recording. This was true when considering all CL1a neurons, and when separating them according to columnar identity (data not shown).

The remaining POL neurons of the central complex fell into three classes: first, tangential neurons of the $\mathrm{PB}$ (TB2 neurons), second, CL1b/d and CL1c/d neurons, and third, conditionally polarization-sensitive columnar neurons (CPU2, CPU4, CL2 neurons). Corresponding to their identical morphology, the $\Phi_{\text {max }}$ values of the 5 recorded TB2 neurons were clearly distinct from randomness and were tightly clustered around a mean vector of $178^{\circ}$ (Fig. 7B) (Rao's spacing test, $\mathrm{U}=226, p<0.01)$. The CL1b/D-and CL1c/d neurons combined with the conditionally polarization-sensitive cells covered a wide range of $\Phi_{\max }$ values (Fig. $7 C$ ) (Rao's spacing test, $\mathrm{U}=82, p>0.95)$. When plotted against the innervated $\mathrm{PB}$ columns, $\Phi_{\max }$ values showed a tendency to cluster along the diagonals of each hemisphere (Fig. $8 B$ ), but this observation was statistically not significant. When the data from this study were combined with the data on 19 columnar neurons from Heinze and Homberg (2007), the new columnar cell types were not distinct from the types described already (Fig. $8 D$ ). The combined data set exhibited a highly significant linear correlation between the $\Phi_{\max }$ values of the neurons and their innervated $\mathrm{PB}$ column, despite the presence of some outliers $(p<0.0001, r=0.67)$. TB2 neurons were located on the same regression line when their varicose arborization domains were plotted against the neuron's $\Phi_{\max }$ values (data not shown).

Polarization-sensitive neurons with input regions in the LAL Several types of columnar cells (types CL1, CP2, CPU1, CPU2) have varicose and, thus, presumably presynaptic terminals in areas of the LAL. We were, therefore, interested to identify potentially postsynaptic neurons in the LAL that might connect the central-complex polarization vision network to descending pathways and motor control circuits of the thorax. Two cell types were found that provide a likely substrate for processing the output signals from the central complex. Both cell types were polarization-sensitive (Fig. 9). The first cell, encountered once, had smooth input arborizations concentrated in the ventral shell of the left LAL and varicose output ramifications extending bilaterally into the posterior protocerebrum (LAL-pPC neuron) (Fig. $9 A$ ). The second neuron [LAL- lateral triangle (LT) neuron] (Fig. $9 B$ ) was analyzed in two recordings from the same hemisphere. It connected large parts of the ipsilateral LAL with the contralateral lateral triangle. In one preparation, additional processes were observed in the median olive (Fig. 9B). The polarity of the neuron 


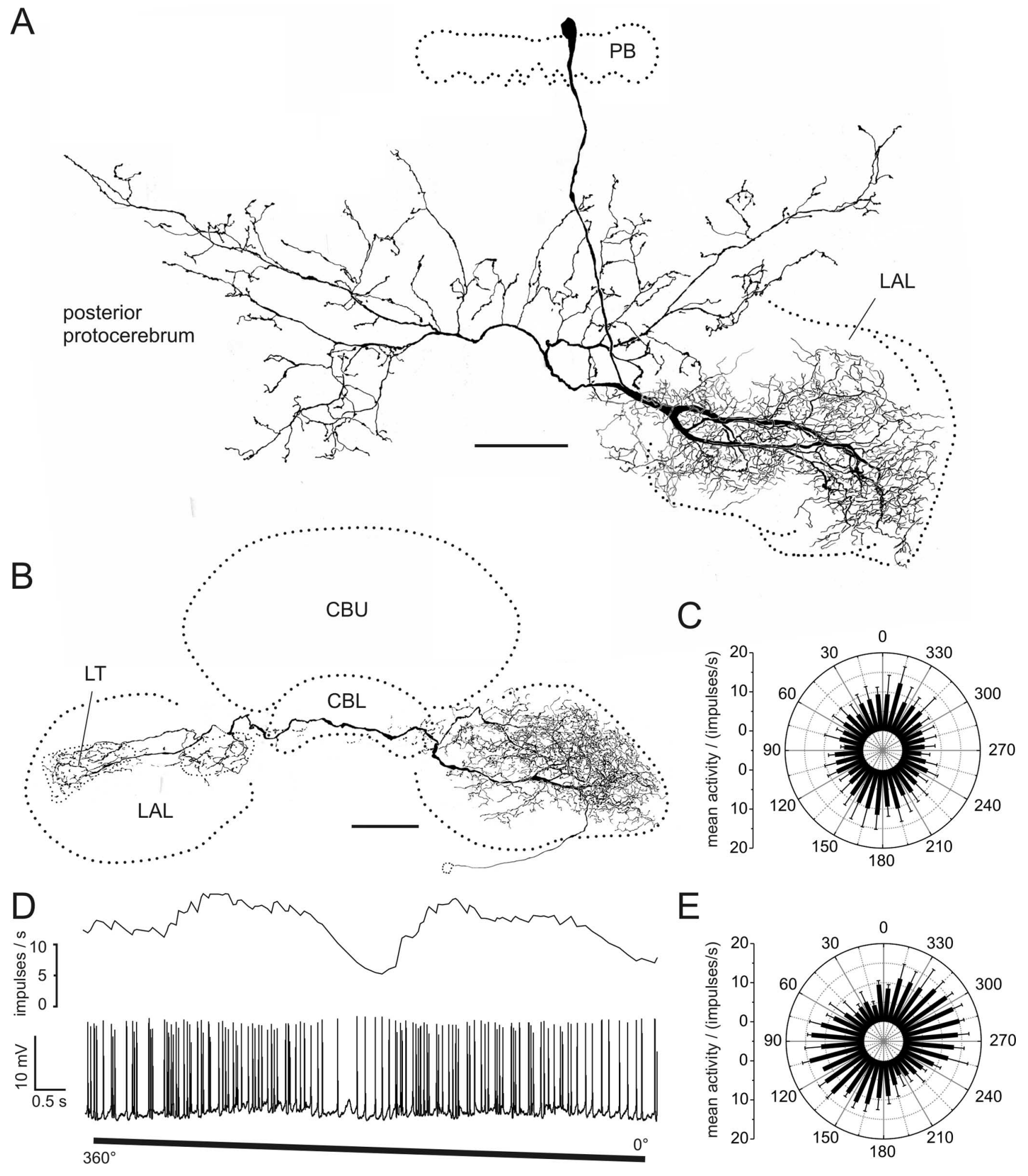

Figure 9. POL neurons with input arborizations in the LAL. A, Frontal reconstruction of a LAL-pPC neuron. The cell has smooth endings in the left LAL. Bilateral projections to the posterior protocerebrum have varicose terminals. $B$, Frontal reconstruction of a LAL-LT neuron. The neuron connects the LAL of the left hemisphere with the LT in the right hemisphere. Side branches are also present in the right median olive. The location of the soma could only be inferred by the course of the faintly stained primary neurite. C, Circular plot of mean spiking frequency from the LAL-pPC neuron shown in $\boldsymbol{A}$ (means $+S D, n=6$, bin size $10^{\circ}, p=4.2 \times 10^{-8}$ ). $\boldsymbol{D}$, Neuronal activity of the LAL-LT neuron presented in $\boldsymbol{B}$ during $360^{\circ}$ rotation of the polarizer. $\boldsymbol{E}$, Mean spiking activity of the LAL-LT neuron presented in $\boldsymbol{B}$ plotted against $E$-vector orientation during four rotations of the polarizer (error bars $=\mathrm{SD}$, bin size $10^{\circ}, p<10^{-12}$ ). $S$ Cale bars, $100 \mu \mathrm{m}$.

was unclear as all endings appeared finely beaded in one preparation and the labeling was incomplete in the second preparation.

Both cell types showed polarization opponency with broad tuning curves (Fig. $9 C-E) . \Phi_{\max }$ values differed widely between the recordings (LAL-pPC: $173^{\circ}$; LAL-LT: $118^{\circ}, 10^{\circ}$ ), whereas the background activity was similar in all cases (LAL-pPC: 10 impulses/s; LAL-LT: 9 and 6 impulses/s). During E-vector rotations LAL-LT neurons reached peak instantaneous frequencies of 43 and 27 impulses per second, the LAL-pPC neuron 70 impulses per second. 


\section{Discussion}

In the desert locust, a variety of neurons of the central complex is sensitive to dorsally presented polarized light, suggesting a key role of this brain area in sky compass orientation (Vitzthum et al., 2002; Homberg, 2004; Heinze and Homberg, 2007). In the present study we have characterized additional elements of the polarization vision network in the central complex. Among these, CL1 neurons are likely to fill the gap between input and output elements and may be importantly involved in generating the topographic representation of $E$-vector orientations in the PB. Neurons of three other cell types were polarization sensitive only in some recordings but not in others, suggesting their conditional recruitment to the polarization vision network. Finally, we identified neurons that are suited to relay polarization vision information to descending pathways. When these data are combined with previously described cell types (Vitzthum et al., 2002; Heinze and Homberg, 2007), a general connectivity scheme of the polarization coding network in the locust central complex emerges (Fig. 10). All major tangential cells of the CBL and the $\mathrm{PB}$, and all columnar cells with ramifications in the $\mathrm{PB}$ are polarization sensitive. In contrast, tangential and pontine neurons innervating the $\mathrm{CBU}$ have never been shown to be polarization-sensitive. These findings suggest that the $\mathrm{CBL}$, together with the $\mathrm{PB}$, processes compass information, whereas the $\mathrm{CBU}$ is involved in other types of sensory coding, which is potentially integrated with compass information via common output neurons.

\section{Connecting the CBL with the $\mathrm{PB}$}

After reaching the lateral triangle and median olive of the LAL, polarized light information is transferred to tangential neurons innervating the CBL (Pfeiffer et al., 2005; Träger et al., 2008). At a later processing stage, columnar and tangential neurons of the $\mathrm{PB}$ are tuned to particular $E$-vector orientations depending on the column they innervate (Heinze and Homberg, 2007). CLla neurons, presented here, are likely to close the gap in information flow between the CBL-input and the polarotopic map in the PB. The polarity of CL1a cells is unique among columnar neurons in the locust central complex and suggests signaling from dendrites in the CBL to axonal outputs in the PB. Presumed input arborizations of CLla neurons are concentrated in dorsal layers of the CBL, coinciding with layers served by TL2 tangential neurons, which are regarded as the dominant polarization-vision input to the CBL and have been also characterized in crickets (Sakura et al., 2008). In contrast, CL1b-d cells are likely to receive input in the PB and convey this information to the CBL. Similar cells of both polarities have been identified also in Drosophila, suggesting common anatomical pathways (Hanesch et al., 1989).

In contrast to the compass-like arrangement of $E$-vector tunings of all other columnar neurons in the PB, $\Phi_{\max }$ values of CL1a neurons were distributed randomly, with highly different $E$-vector tunings occurring in the same $\mathrm{PB}$ column. As this tuning behavior is more similar to input neurons of the central complex than to the remaining columnar cells, it supports the hypothesis that CL1a neurons represent an earlier processing stage than the polarotopic columnar neurons. Nevertheless, it raises the question why neurons within identical columns behave differently between preparations. $\Phi_{\max }$ values of CL1a neurons are either influenced by unknown parameters, or the population of these neurons consists of more than one cell per column. The latter may obscure potential column-dependencies of $\Phi_{\max }$ values, if e.g., siblings of CLla neurons differ by $90^{\circ}$ in their $E$-vector tuning. No evidence was found for the hypothesis that $\Phi_{\max }$ values of CLla neurons depend on daytime.
CL1b-d neurons have a polarity corresponding to other columnar neurons, based on structure of terminals and the occurrence of large PSPs in the PB. Their wiring scheme is identical to that of CL1a cells, which allows for complex bidirectional information flow between the CBL and the PB. Although CL1 cells constitute the potential neuronal substrate, it remains a significant challenge to explain the emergence of the ordered polarotopic $E$-vector representation from an apparently random distribution of $E$-vector tunings.

\section{Column-specific cell types}

Heinze and Homberg (2007) demonstrated a compass-like topography of $E$-vector tunings in the varicose arborization domains of TB1 tangential neurons of the PB. Their morphology is similar, but not identical to the newly described TB2 cells. $\Phi_{\max }{ }^{-}$ Values of TB2 neurons differed by $90^{\circ}$ from the values of TB 1 cells arborizing in the same columns (Heinze and Homberg, 2007). TB2 cells were only found with varicose arborizations in the inner- and outermost PB columns, suggesting that there is no isomorphic set of these cells covering the whole PB. Specific innervation of the outer- and innermost central-complex columns has also been shown for other cell types. Analysis of citrulline immunostaining revealed 16 PU1 pontine neurons (one neuron per column) plus two additional neurons, which specifically connect the inner- and outermost columns of the same hemisphere (T. Siegl and U. Homberg, unpublished observations). Likewise, the presumed connectivity scheme of CPU1a neurons suggests that the outermost PB columns are connected differently from all other columns. In Drosophila, the exceptional projections of the outermost columns of the horizontal fiber system were interpreted to underlie a particular optomotor response. Flies turn toward objects in the frontal field of view, but turn away from objects in the rear field (Mronz and Strauss, 2008). Assuming that the width of the PB encodes azimuthal space around the animal (Strauss, 2002a; Heinze and Homberg, 2007), the outermost columns of the PB might encode the rear part of the visual field. Specialized neurons with inverted wiring and response characteristics in these columns might trigger the repulsive motor reaction in contrast to the frontally attractive stimulus. Whether similar behavioral findings also apply to the locust remains to be shown.

\section{Conditional polarization sensitivity}

All neurons of the polarization vision system of the locust, described so far, showed robust responses to polarized light (Vitzthum et al., 2002; Pfeiffer et al., 2005; Heinze and Homberg, 2007; Kinoshita et al., 2007; Pfeiffer and Homberg, 2007). In contrast, three cell types presented here (CPU2, CL2, and likely CPU4) were polarization sensitive or polarization blind, depending on the individual preparation. This variability in responses was clearly not caused by varying recording quality or tissue damage, because these variations also occurred in all other recordings. In the polarization-sensitive CPU2- and CL2 neurons, background activity was higher than in the nonresponding units. Furthermore, responses to unpolarized light stimuli were only present in the polarization-blind CPU2- and CPU4 units. Both findings indicate that the source of synaptic input differs between the polarization-sensitive and the polarization-blind state of the neurons. Conditionally polarization-sensitive neurons may, therefore, be recruited to the polarization vision network depending on the internal state of the animal and its behavioral context.

The central complex has a dual role as a sensory processing and a motor control center (Strausfeld, 1999; Strauss, 2002b; Homberg, 2008). Context dependent changes in network prop- 

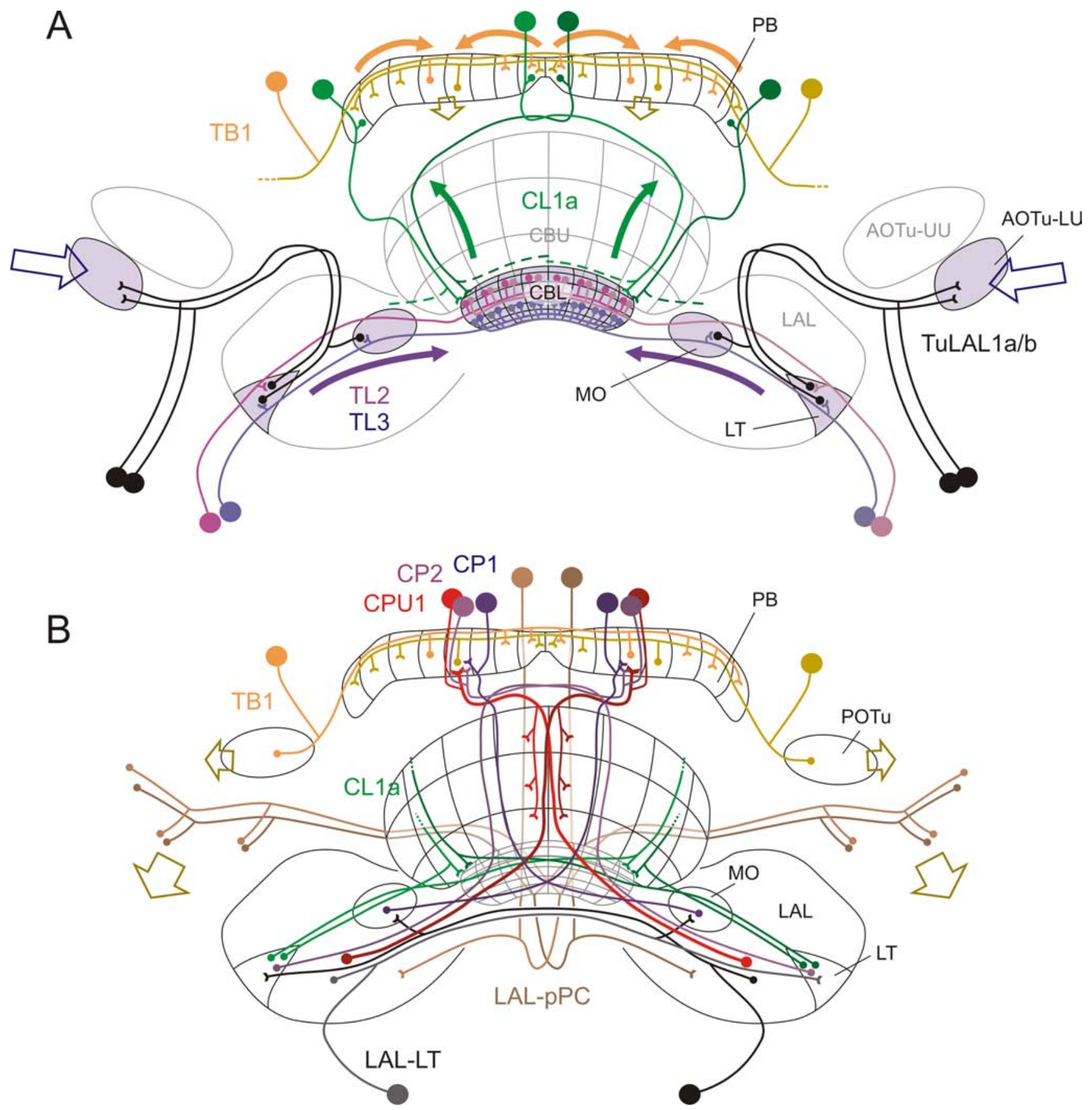

Figure 10. Proposed scheme of the polarization coding network in the central complex. $A$, Input pathways. Polarization vision information is transferred from the optic lobe to the lower unit of the anterior optic tubercle (AOTu-LU; open blue arrows) and reaches the LT and median olive (MO) via TuLAL1a/b neurons (Pfeiffer et al., 2005). TL2 and TL3 tangential neurons connect the LT and M0 to different layers of the CBL (Träger et al., 2008). CL1a neurons are candidates to transmit polarization vision signals from the CBL to the PB. For clarity, this connectivity is only shown for one double column in each hemisphere (CL1a axons to the LT have been omitted). TB1 neurons integrate the signals within the PB and are the first neurons that contribute to the topographic representation of $E$-vectors in the PB columns (shown for two TB1 cells only) (Heinze and Homberg, 2007). Open, brown arrows indicate the columnar output of the two TB1 neurons. $B$, Output pathways. The output from TB1 neurons in the PB is likely transferred onto CPU1, CP1, and CP2 columnar neurons projecting to the lateral accessory lobes (LALs, red), the MO (blue), or the LT (violet; Heinze and Homberg, 2007). Of these, (PU1 neurons receive additional input in the CBU. A second pathway connects the CBL directly to the LT via small-diameter axons of CL1a/b neurons, not involving the PB. All terminals of columnar neurons within the LT and MO could potentially provide input to LAL-LT neurons (black), which connect these areas with the contralateral LAL. This neuron type, as well as CPU1 neurons, might synapse onto the LAL-pPC neuron, which provides a connection to the posterior protocerebrum. TB1 neurons provide another possible output from the PB to the posterior optic tubercle (POTu). Descending neurons might receive input from either of these regions (brown, open arrows), and/or from the LAL, to provide information flow to motor control circuits in the thorax. AOTu-UU, Upper unit of the anterior optic tubercle.

erties and neural activities are, therefore, to be expected. Only a fraction of sensory channels that continuously provide the animal with information about the outside world is relevant at any time, depending on the behavioral context (mating, feeding, migrating, escaping, etc.). In locusts, stationary flight is correlated with dramatic activity changes in neurons of the central complex and LAL (Homberg, 1994). In Drosophila, learning of visual objects and landmark parameters is associated with neurons of the central complex (Liu et al., 2006; Neuser et al., 2008). These findings suggest considerable learning- and behavior-dependent 
plasticity in the central complex. Recruitment of neuronal assemblies in motor control networks has been reported in insects (Reichert, 1993; Staudacher and Schildberger, 1998) and in vertebrates (Li et al., 2007). Obligatory POL neurons might, therefore, be part of a core network underlying general azimuth coding, whereas conditional POL neurons might be recruited only when a particular navigational task is performed. Interestingly, two of the three conditional POL neurons innervate the noduli. Evolutionarily, the noduli are the most recent additions to the central complex and may be associated with the origin of flight (Homberg, 2008). Especially large noduli are present in flies (Strausfeld, 1999), suggesting that they provide additional computational power during complex flight maneuvers.

\section{Beyond the central complex}

The LAL-LT and LAL-pPC neurons are likely postsynaptic to the central-complex network. The LAL-LT neuron communicates between projection areas of columnar neurons across the brain midline, whereas the LAL-pPC neuron transfers polarization information to the posterior protocerebrum. Both the LAL and the posterior protocerebrum are regions where many descending neurons receive their input (Williams, 1975; Strausfeld and Seyan, 1985; Staudacher and Schildberger, 1998; Okada et al., 2003). The different $\Phi_{\max }$ values of the two LAL-LT recordings implicate that several LAL-LT neurons differ in E-vector tuning or that $E$-vector tuning in the same neuron is dynamically adjusted corresponding to motor demands. Transforming polarization vision signals into motor commands clearly requires statedependent gating and plasticity demonstrated at various levels of motor control (Rowell and Reichert, 1986; Reichert, 1993; Staudacher and Schildberger, 1998). These mechanisms will have to be taken into account when further analyzing the role of polarized skylight in directional steering in insects.

\section{References}

Batschelet E (1981) Circular statistics in biology. London: Academic.

Clements AN, May TE (1974) Studies on locust neuromuscular physiology in relation to glutamic acid. J Exp Biol 60:673-705.

Eggers A, Gewecke M (1993) The dorsal rim area of the compound eye and polarization vision in the desert locust (Schistocerca gregaria). In: Sensory systems of arthropods (Wiese K, Gribakin FG, Popov AV, Renninger G, eds), pp 101-109. Basel: Birkhäuser.

Hanesch U, Fischbach KF, Heisenberg M (1989) Neuronal architecture of the central complex in Drosophila melanogaster. Cell Tissue Res 257:343-366.

Heinze S, Homberg U (2007) Maplike representation of celestial E-vector orientations in the brain of an insect. Science 315:995-997.

Heinze S, Homberg U (2008) Neuroarchitecture of the central complex of the desert locust: intrinsic and columnar neurons. J Comp Neurol 511:454-478

Homberg U (1994) Flight-correlated activity changes in neurons of the lateral accessory lobes in the brain of the locust Schistocerca gregaria. J Comp Physiol [A] 175:597-610.

Homberg U (2004) In search of the sky compass in the insect brain. Naturwissenschaften 91:199-208.

Homberg U (2008) Evolution of the central complex in the arthropod brain with respect to the visual system. Arthropod Struct Dev 37:347-362.

Homberg U, Paech A (2002) Ultrastructure and orientation of ommatidia in the dorsal rim area of the locust compound eye. Arthropod Struct Dev 30:271-280.

Horváth G, Varjú D (2004) Polarized light in animal vision. Springer: Berlin.

Kinoshita M, Pfeiffer K, Homberg U (2007) Spectral properties of identified polarized-light sensitive interneurons in the brain of the desert locust Schistocerca gregaria. J Exp Biol 210:1350-1361.
Labhart T, Meyer EP (2002) Neural mechanisms in insect navigation: polarization compass and odometer. Curr Opin Neurobiol 12:707-714.

Li WC, Sautois B, Roberts A, Soffe SR (2007) Reconfiguration of a vertebrate motor network: specific neuron recruitment and contextdependent synaptic plasticity. J Neurosci 27:12267-12276.

Liu G, Seiler H, Wen A, Zars T, Ito K, Wolf R, Heisenberg M, Liu L (2006) Distinct memory traces for two visual features in the Drosophila brain. Nature 439:551-556.

Mappes M, Homberg U (2004) Behavioral analysis of polarization vision in tethered flying locusts. J Comp Physiol A Neuroethol Sens Neural Behav Physiol 190:61-68.

Mronz M, Strauss R (2008) Visual motion integration controls attractiveness of objects in walking flies and a mobile robot. In: IEEE/RSJ International conference on intelligent robots and systems. Conference proceedings (Chatila R, Kelly A, Merlet J, eds), pp 3559-3564. New York: IEEE.

Müller M (1997) Anatomische und funktionelle Charakterisierung der unteren Einheit des Zentralkörpers im Gehirn der Heuschrecke Schistocerca gregaria. $\mathrm{PhD}$ thesis, Universität Regensburg.

Müller M, Homberg U, Kühn A (1997) Neuroarchitecture of the lower division of the central body in the brain of the locust (Schistocerca gregaria). Cell Tissue Res 288:159-176.

Neuser K, Triphan T, Mronz M, Poeck B, Strauss R (2008) Analysis of a spatial orientation memory in Drosophila. Nature 453:1244-1247.

Okada R, Sakura M, Mizunami M (2003) Distribution of dendrites of descending neurons and its implications for the basic organization of the cockroach brain. J Comp Neurol 458:158-174.

Pfeiffer K, Homberg U (2007) Coding of azimuthal directions via timecompensated combination of celestial compass cues. Curr Biol 17:960-965.

Pfeiffer K, Kinoshita M, Homberg U (2005) Polarization-sensitive and light-sensitive neurons in two parallel pathways passing through the anterior optic tubercle in the locust brain. J Neurophysiol 94:3903-3915.

Reichert H (1993) Sensory inputs and flight orientation in locusts. Comp Biochem Physiol 104:647-657.

Rowell CH, Reichert H (1986) Three descending interneurons reporting deviation from course in the locust. II. Physiology. J Comp Physiol [A] 158:775-794.

Sakura M, Lambrinos D, Labhart T (2008) Polarized skylight navigation in insects: model and electrophysiology of e-vector coding by neurons in the central complex. J Neurophysiol 99:667-682.

Staudacher E, Schildberger K (1998) Gating of sensory responses of descending brain neurones during walking in crickets. J Exp Biol 201:559-572.

Strausfeld NJ (1999) A brain region in insects that supervises walking. Prog Brain Res 123:273-284.

Strausfeld NJ, Seyan HS (1985) Convergence of visual, haltere, and prosternal inputs at neck motor neurons of Calliphora erythrocephala. Cell Tissue Res 240:601-615.

Strauss R (2002a) Die übergeordnete Steuerung des Laufverhaltens durch das Insektengehirn, studiert mit Methoden der Drosophila-Neurogenetik. Habilitationsschrift, Universität Würzburg.

Strauss R (2002b) The central complex and the genetic dissection of locomotor behaviour. Curr Opin Neurobiol 12:633-638.

Träger U, Wagner R, Bausenwein B, Homberg U (2008) A novel type of microglomerular synaptic complex in the polarization vision pathway of the locust brain. J Comp Neurol 506:288-300.

Vitzthum H, Müller M, Homberg U (2002) Neurons of the central complex of the locust Schistocerca gregaria are sensitive to polarized light. J Neurosci 22:1114-1125.

von Frisch K (1949) Die Polarisation des Himmelslichtes als orientierender Faktor bei den Tänzen der Bienen. Experientia 5:142-148.

Wehner R (1984) Astronavigation in insects. Annu Rev Entomol 29:277-298.

Wehner R, Labhart T (2006) Polarization vision. In: Invertebrate vision (Warrant E, Nilsson DE, eds), pp 291-348.Cambridge: Cambridge UP.

Williams JLD (1975) Anatomical studies of the insect central nervous system: a ground-plan of the midbrain and an introduction to the central complex in the locust, Schistocerca gregaria (Orthoptera). J Zool (Lond) 176:67-86. 